


\section{SPECIAL SESSION ON ADVANCED MODULATION FORMAT}

Phase-modulated OOK formats at 40G and 160G [6353-12]

Y. Su, L. Möller

Cost-effective optical duobinary transmitters [6353-13]

H. Kim, H. Lee, S. Kim, G. Lee, S. Hwang, Y. Oh

\section{NGN AND STUDENT PAPER AWARD CONTEST}

Wavelength- and time-division reconfigurable optical add/drop multiplexer (ROADM) using a time-space conversion and a MEMS optical switch [6353-15]

R. Itoh, T. Konishi, K. Itoh

Performance of the OSNR monitoring technique based on improved polarization-nulling method [6353-16]

H. Choi, J. Lee, S. Jun, S. Shin, Y. Chung

Control of EDFA gain transient in optical burst network using burst control packet channel [6353-17]

J. Cho, P. Park, M. Cho, Y. Won

All-optical analog-to-digital conversion using optical interconnection for gray code coding [6353-18]

T. Nishitani, T. Konishi, K. Itoh

\section{ELECTRONIC SIGNAL PROCESSING}

FEC assembly at ingress node in labeled optical burst switching system [6353-23]

Y. Xu, J. Wu, K. Xu, J. Lin

\section{OXC, OADM, AND OPM}

A novel algorithm based on the dynamic threshold for the wavelength assignment in WDM networks [6353-25]

M. Fu, Z. Le, W. Dong, H. Zhu

An optimal policy based on the genetic algorithm for the dynamic threshold of the optical network [6353-26]

H. Zhu, Z. Le, W. Dong, M. Fu

A low optical-coherent-crosstalk thin-film filter-based 1x2 fiber-optic add-drop module in a doublereflection configuration [6353-27]

S. Sumriddetchkajorn, K. Chaitavon

Thin-film filter-based wavelength division multiplexers/demultiplexers in an angle-multiplexed architecture [6353-28]

S. Sumriddetchkajorn, K. Chaitavon

Fast online simultaneous monitoring of PMD and chromatic dispersion with reduced polarization dependent gain [6353-29]

G. Ning, P. Shum, S. Aditya, Y. Gong

Experimental research of obtaining DGD from DOP ellipsoids [6353-30]

G. Duan, B. Yang, X. Zhang

The compensation performance of different modulation formats in polarization mode dispersion systems [6353-32]

G. Duan, B. Yang, X. Zhang

Comparison of different modulation formats for polarization mode dispersion compensators [6353-33]

H. Wu, L. Chen, S. Wen 


\title{
Control of EDFA gain transient in optical burst network using burst control packet channel
}

\author{
Jeong Sik Cho*a , Paul K. J. Park ${ }^{\mathrm{b}}$, Min Jae Cho ${ }^{\mathrm{a}}$, Yong Hyub Won ${ }^{\mathrm{a}}$ \\ ${ }^{a}$ School of Engineering, Information and Commutations University, 119 Munijiro, Yuseong-gu, \\ Daejeon, Korea, 305-714 \\ bamsung Advanced Institute of Technology, San 14-1, Nongseo-ri, Giheung-eup, Yongin-si, \\ Gyeonggi-do, Korea, 449-712
}

\begin{abstract}
We propose a control technique for erbium doped fiber amplifier (EDFA) gain transient occurring in optical burst network. In the optical burst network, optical packets are generated randomly and packet stream has idle time when no packet exists. This bursty nature of optical burst network causes gain transient in the EDFA with slow gain dynamics. To resolve this problem, the optical power of a channel delivering the burst control packets (BCPs) is modulated based on the information of the burst data (BD) packets contained in the BCP itself to feed constant power to the EDFA. We experimentally demonstrate $2.0-\mathrm{dB}$ and $2.6-\mathrm{dB}$ reductions in power fluctuation and gain transient in EDFA output, respectively. We show the stable transmission of $2.488 \mathrm{Gbit} / \mathrm{s}$ rate $\mathrm{BCP}$ and $9.953 \mathrm{Gbit} / \mathrm{s}$ rate $\mathrm{BD}$ packets using the proposed technique. The proposed method can be applied to optical burst switching (OBS) network which adopt out-ofband control channel covering small number of BD channels.
\end{abstract}

Keywords: EDFA, gain transient control, OBS, burst control packet

\section{INTRODUCTION}

As Internet traffic increased, the optical network as the infrastructure of modern network grew to deliver more and more Internet protocol (IP) based packets. The representative feature of IP traffic is that it emerges randomly, in other words, it is bursty. In order to accommodate the IP traffic, the optical network must support fast assembling data into a packet and safe delivery of the packet based on fast transmission, reception and switching, etc. Optical burst switching (OBS) has been studied as a practical technique to implement packet based optical network before reaching eventual highspeed optical packet switching (OPS) network [1]. In the OBS network, data packets are assembled into long bursts of a few microseconds to hundred microseconds and transported through a core network. The burst data streams have idle intervals, no optical power period, which cause abrupt power change in input to the erbium doped fiber amplifiers (EDFAs) deployed in the optical links. In this case, gain transient appears as a result of slow gain dynamics of the EDFA. It means that the EDFA can not fast respond to the abrupt power change of incoming burst packet and it needs more or less long time comparable to the length of burst packet. Thus, the EDFA show slow gain adaptation, so called, gain transient. And the transient induces inter- and intra-packet power variation and requires a special technique to resolve it or the optical receiver with high dynamic range. Previously proposed techniques for overcoming the transient utilized all optical feedback or all-optical gain clamping [2], inversely modulated additional channel [3], constant-wave channel [4], and packet envelope detection technique [5]. However, these approaches suffer from relaxation oscillation in output packet [6] or require high power optical source [4], and result in waste of wavelength resource [3-5].

In this paper, we propose and experimentally demonstrate a gain control technique using burst packet information, such as burst size and offset time, delivered by burst control packet (BCP). The optical power of the BCP channel is adjusted based on the packet information in the BCP itself and it compensates the power variation in burst data (BD) channels to feed constant power to an EDFA. This technique differs from previous signal feed-back approaches $[1,4]$ because it forward feeds the control signal as like ref. [2]. Moreover, it can effectively utilize wavelength resource because this technique utilizes not a dummy channel or wavelength but the BCP channel for gain transient control. The EDFA fed by constant input power shows no gain or power transient in output signal.

Optical Transmission, Switching, and Subsystems IV, edited by

Chang Soo Park, Shizhong Xie, Curtis R. Menyuk, Ken-ichi Kitayama,

Proc. of SPIE Vol. 6353, 63530G, (2006) · 0277-786X/06/\$15 - doi: 10.1117/12.691701

Proc. of SPIE Vol. 6353 63530G-1 
Even though the modulation of BCP channel can resolve the gain transient problem in EDFA link, it could induce side effect such as signal distortion or performance degradation in BCP channel. We discuss this issue and experimentally examine this. And the expected problems in application of the proposed technique are discussed.

\section{TRANSIENT CONTROL TECHNIQUE}

In the OBS network, the BCPs are transmitted prior to the BD packets as much as offset time interval [1]. At the edge and core nodes, the BCP is processed to identify the next destination of assembled and incoming BD packet and prepare punctual switching operation. This operation was successfully demonstrated and reported in ref. [7]. The BCP processor well knows the size of the BD packet and controls a switch fabric based on the information in the BCP. Thus, the exact envelope information of every BD packet incoming to and outgoing from a node can be extracted from the BCP processor. This information is used for generating control signal to modulate the power of the BCP channel. And the envelope of the BCP channel has reverse image of the total power of the BD channels as shown in Fig. 1.

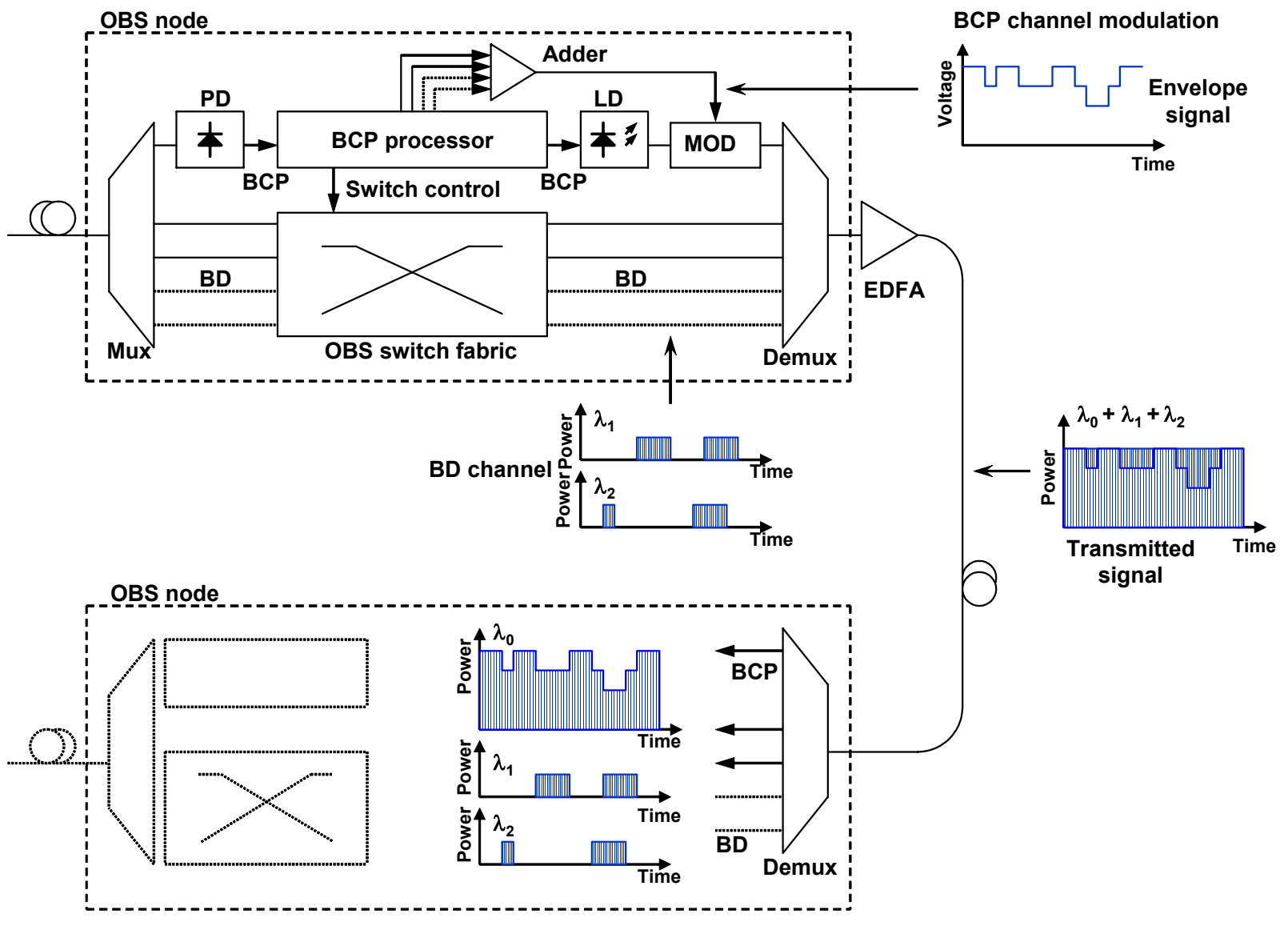

Fig. 1. Schematic diagram of the proposed gain transient control technique (PD: Photodetector, LD: Laser diode, MOD: External electro-optic modulator).

The total power of all BD packets is calculated by analog adder circuit made of operational amplifier. The analog adder generates the electric signal corresponding to the sum of inverted envelope for total power of BD and direct current 
(DC) bias. The DC bias signal in the analog adder controls the modulation depth of the BCP channel and determines biasing point of an external electro-optic modulator. And the electric signal is fed into the modulator, then, the amplitude of the $\mathrm{BCP}$ channel is adjusted according to the existence of $\mathrm{BD}$ packet. This $\mathrm{BCP}$ channel is combined with BD channels. Due to this process, the idle period between two BD packets is compensated by the power of BCP channel. Then, the optical BCP signal with constant total power is fed into the modulator EDFA. As a result, the EDFA gain transient can be effectively suppressed by the proposed technique. At the next OBS node, the BCP and BD channels are demultiplexed and the $\mathrm{BCP}$ goes to the $\mathrm{BCP}$ processor. Then, the $\mathrm{BCP}$ processor generates new $\mathrm{BCP}$ which has varying optical power according to the existence of the BD packets.

In order to compensate the missing optical power between two BD packets, the BCP channel must not have any idle periods. That is, the $\mathrm{BCP}$ channel must transmit packet stream continuously. To sustain continuous stream in the $\mathrm{BCP}$ channel, it could be composed of meaningful BCPs and dummy BCPs by time division multiplexing method [7].

In the proposed method, the $\mathrm{BCP}$ channel is modulated. Thus, the performance degradation in the $\mathrm{BCP}$ channel is expected. In the next part, this degradation effect is examined and the maximum value of modulation depth is investigated. Also, the architecture for allocation of the BCP and BD channels is discussed.

\section{EXPERIMENTS AND RESULTS}

An experimental setup for the proposed technique is shown in Fig. 2.

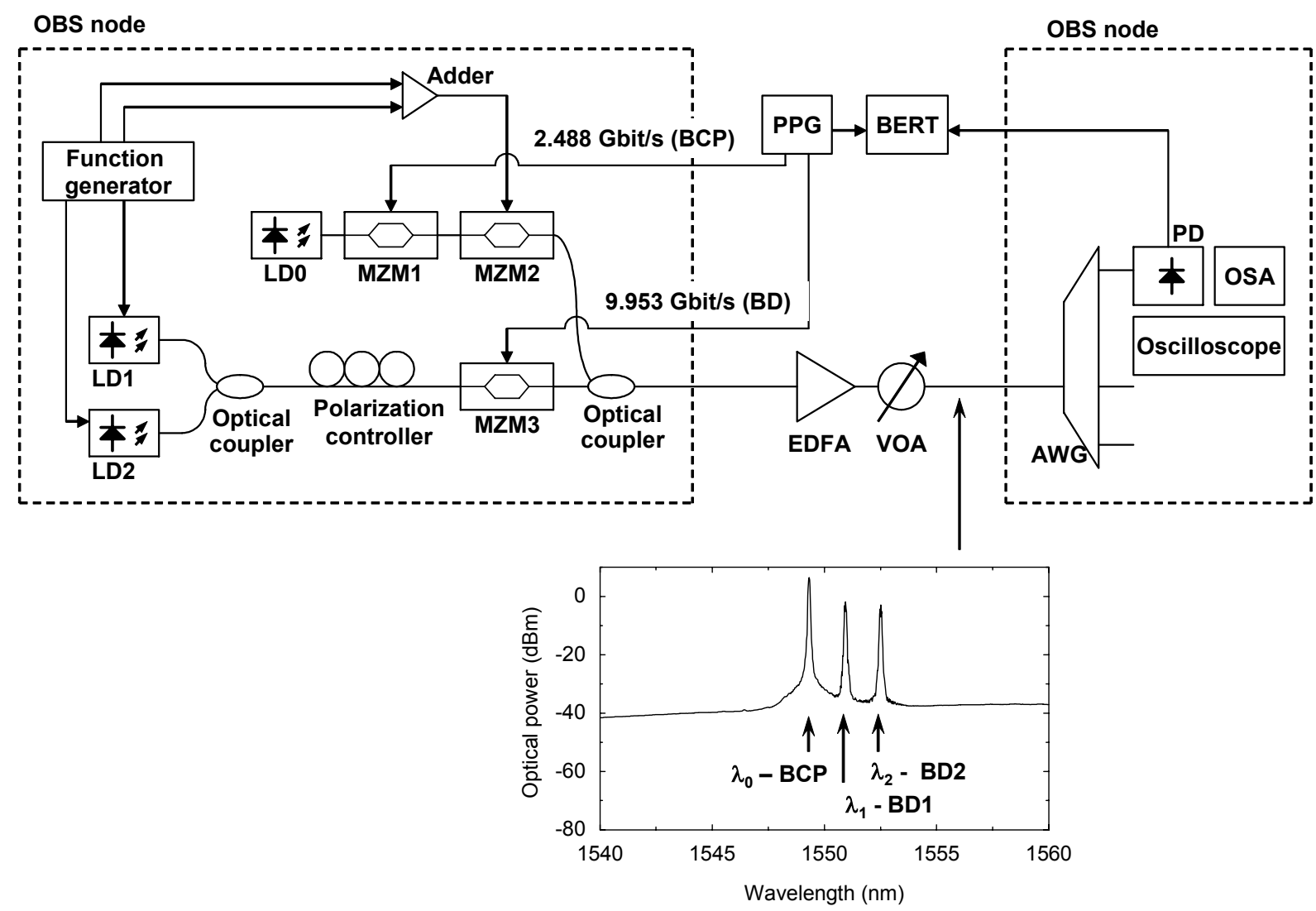

Fig. 2. Experimental setup (PPG: Pulse Pattern Generator, BERT: Bit Error Rate Tester, OSA: Optical Spectrum Analyzer) and optical spectrum of EDFA output. 
The laser diode 0 (LD0) at the wavelength $\lambda_{0}, 1549.32 \mathrm{~nm}$, was modulated at $2.488 \mathrm{Gbit} / \mathrm{s}\left(2^{31}-1 \mathrm{PRBS}\right)$ using the Mach-Zehnder modulator1 (MZM1) for the BCP channel. And this BCP channel was further modulated by inversed envelope with respect to the total envelope of all BD channels generated by the signal adder using the MZM2. For the BD1 and BD2 channels, LD1 $\left(\lambda_{1}=1550.92 \mathrm{~nm}\right)$ and LD2 $\left(\lambda_{2}=1552.52 \mathrm{~nm}\right)$ were turned on and off by the control signal from a function generator (FG, TTi - TGA1244) to emulate BD arrival and control signal generation after BCP processing. For the convenience of experiment, the BCP processor was replaced by the FG but the same results can be obtained for the field programmable gate array (FPGA) based BCP processor [7]. The two beams were optically combined by $3-\mathrm{dB}$ optical coupler and modulated at $9.953 \mathrm{Gbit} / \mathrm{s}\left(2^{31}-1\right.$ PRBS) using the MZM3. The BCP and BD packets were combined and passed through an EDFA of 15-dB gain. The total input power was $-8 \mathrm{dBm}$. The output spectrum of the EDFA is shown in Fig. 2. The power of the BCP channel was far bigger than those of the BD channels. To emulate long transmission link and exclude transmission penalty, a variable optical attenuator (VOA) attenuated the signal in $15 \mathrm{~dB}$. The signals were demultiplexed by an arrayed waveguide grating (AWG) and detected by a photodetector (PD).

In the experiment, the MZM (MZM2) was used for the control of optical power of the BCP channel. But it shows nonlinear transfer function so that it can be replaced a liner modulator such as an electro-absorption modulator (EAM), etc. Nevertheless, the MZM did not show any critical problem in the experiment.

The input and output streams for the EDFA are shown in Fig. 3.

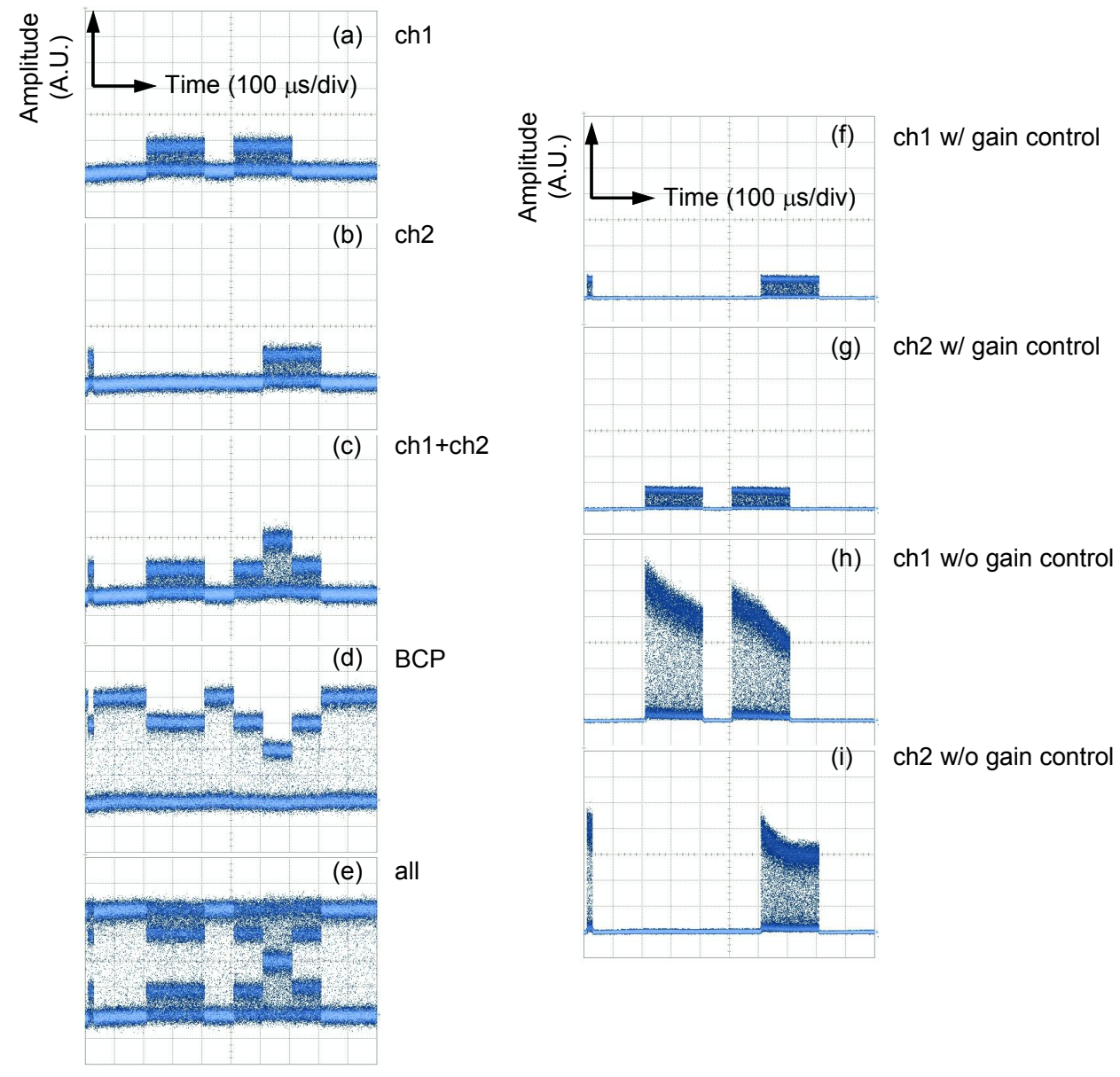

Fig. 3. Timing diagram for (a, b) BD channels before amplification, (c) a combined signal of BD channels, (d) the BCP channel, (e) a combined signal of all BD channels and BCP channel, (f, g) BD channels after amplification without gain control, and $(h, i)$ the gain controlled outputs of BD channels from an EDFA 
For the BD1, two 200- $\mu$ s length burst packets were generated and $20-\mu$ s and $200-\mu$ s length burst packets were generated for the BD2 by the FG. To examine the coexistence condition for BD1 and BD2, two packets of each channel were allocated to be partially overlapped. Power excursion by gain transient was maximally $2.0 \mathrm{~dB}$ for the EDFA output of the $\mathrm{BD} 1$ and $\mathrm{BD} 2$. And packet by packet power fluctuation was $2.6 \mathrm{~dB}$ in maximum. For transient controlled condition, the results show a little power excursion $(0.3 \mathrm{~dB})$ in output burst packets. This means that the proposed technique can effectively suppress the gain transient of an EDFA.

In the proposed technique, the optical power of the BCP channel is changed when the BD packet exists. Thus, the variation of power can cause transmission errors in the BCP channel. The power variation can be described by the modulation index, $\delta$, defined by

$$
\delta=\frac{V_{\max }-V_{\min }}{V_{\max }+V_{\min }}
$$

where the $V_{\max }$ and $V_{\min }$ are the maximum and minimum values of detected BCP signal in voltage unit, respectively. For a conventional DC-coupled receiver of $2.5 \mathrm{Gbit} / \mathrm{s}$, the minimum value of modulation index was about 0.3 in our experiment. For the alternating current (AC)-coupled receiver, this value could be improved further up to 0.4 as shown in Fig. 4. But, further increase in modulation index was not achieved because of severe penalty for deeper modulation. Higher modulation index means that more BD channels can be controlled by one $\mathrm{BCP}$ channel. However, the optical power of the LD for BCP generation must be increased to support a number of BD channels. Thus, the number of BD channels covered by a BCP channel is limited.

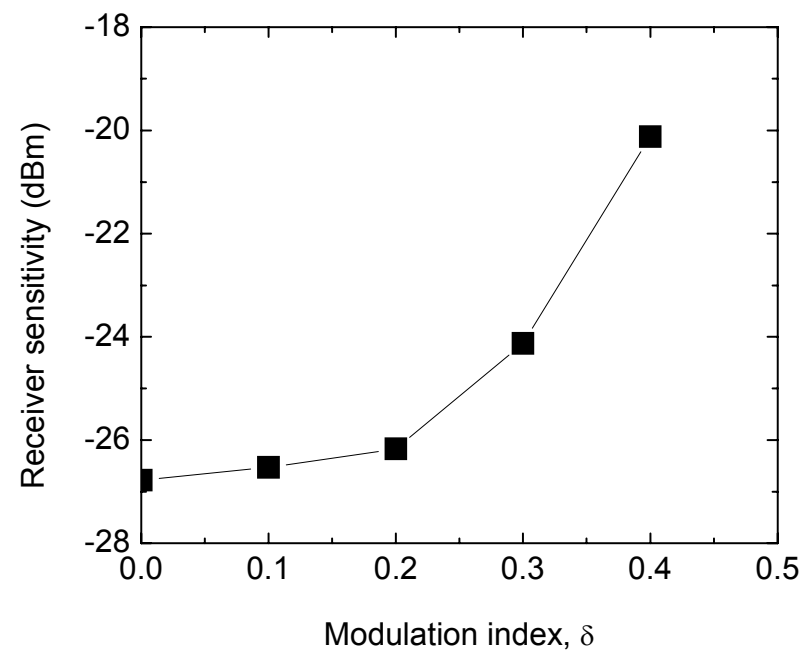

Fig. 4. Sensitivity of AC coupled receiver for various modulation index at a BER of $1.0 \times 10^{-9}$

In the proposed method, out-of-band BCP channel is used for BCP delivering and gain transient control. The BCP could be transported by in-band or out-of-band method. In the case of OBS network, the out-of-band method has been generally considered and studied [1]. Out-of-band transmission of BCP has an advantage of easy extracting of the BCP from incoming optical signal. But, if the wavelength difference of the $\mathrm{BCP}$ and the $\mathrm{BD}$ channel increases, the timing relation between the $\mathrm{BCP}$ and the $\mathrm{BD}$ (off-set time) could be broken because of dispersion effect. For instance, $100 \mathrm{GHz}$ spacing (approximately $0.8 \mathrm{~nm}$ near $1550 \mathrm{~nm}$ ) between the $\mathrm{BCP}$ and $\mathrm{BD}$ packet induces about $22 \mathrm{ps}$ delay, when the two packets are transported in 100km via the commercial single mode fiber (SMF), Corning SMF-28 [8]. In the case of $1 \mathrm{THz}$ spacing $(8 \mathrm{~nm})$, the delay increases up to about $0.22 \mathrm{~ns}$. Thus, as difference between the BCP and BD channel is larger, the probability of non-punctual operation of the optical switch fabric increases because of the addition of 
uncertainty to the predetermined offset time. Therefore, the BCP and BD channel must be closely allocated in spectral domain, that is, it is difficult that one $\mathrm{BCP}$ channel delivers lots of $\mathrm{BCP}$ corresponding to several tens of BD channels.

In addition, regarding the modulation index aspect, one BCP channel can not cover large number of BD channels because power ratio of BD channel to BCP channel could be very small as the number of BD channel increases. It results that the signal to noise ratio (SNR) of the $\mathrm{BD}$ channel decreases and power budget of the $\mathrm{BD}$ channel shows small margin between transmitted power and received power. To accommodate a number of BD channel, the modulation index could be increased, however, the receiver sensitivity must be considered in this case as it is shown in Fig. 4.

Finally, if one BCP channel covers large number of BD channels, then the probability of congestion in the BCP channel increases [9]. The BCP could be missed so that the related BD packet could be lost. To prevent this unexpected event, the offset time must be dynamically controlled or a buffer system must be adopted.

Thus, the number of the BD channel covered by one BCP channel has to be examined and carefully considered in system design process. In the calculation, when the number of $\mathrm{BD}$ channel is 10 and the duty of the $\mathrm{BD}$ packets for all $\mathrm{BD}$ channel equals to $10 \%$, the power ratio is $-17 \mathrm{~dB}$ and the optical SNR of the $\mathrm{BD}$ channel is degraded as much as $27 \mathrm{~dB}$ for the $\mathrm{BCP}$ channel. These are too severe for the $\mathrm{BD}$ channel performance. Therefore, the number of $\mathrm{BD}$ channel for a $\mathrm{BCP}$ channel must be optimized in the range of less than 10 .

The BER curves of the BCP channel were measured as shown in Fig. 5. Even for simultaneous operation of BD1 and $\mathrm{BD} 2$, the power penalty was $2.3 \mathrm{~dB}$ and it is less than the dynamic range of $\mathrm{AC}$-coupled receiver $(-27 \mathrm{dBm}$ to $-10 \mathrm{dBm})$. The insets in Fig. 5 show unfamiliar eye diagrams. Especially, in case of two BD channels compensation, the modulation index of 0.33 is clearly presented.

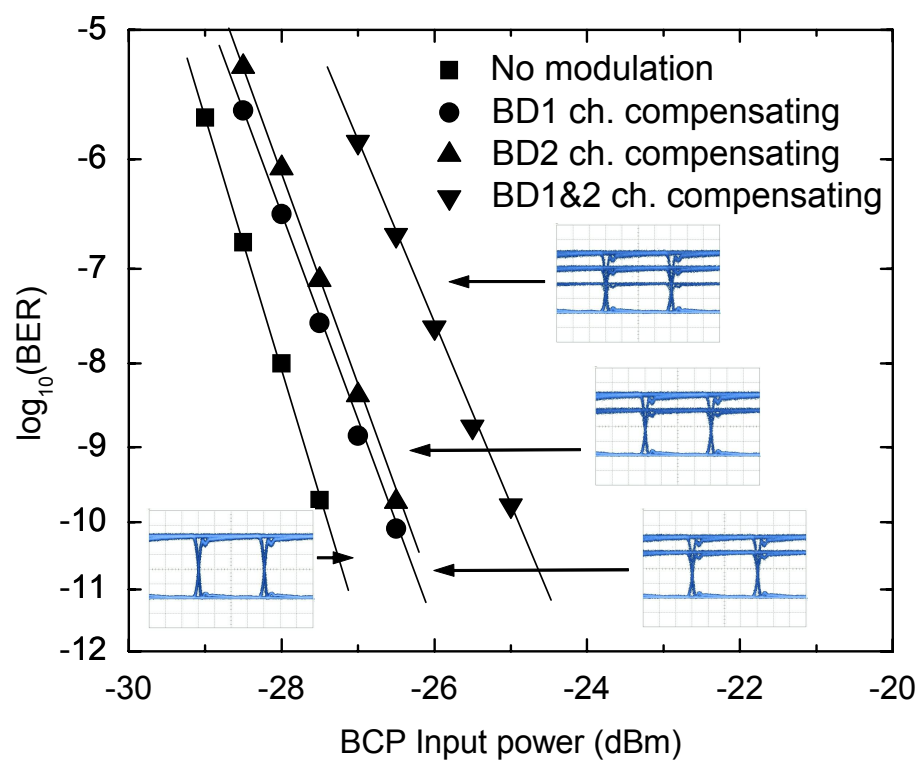

Fig. 5. BER curves of the BCP channel for supporting no BD channel, one BD channel, and two BD channels

Fig. 6 shows two BER curves of the BD1 for the case of with and without gain control. Without the proposed gain control technique, $0.4 \mathrm{~dB}$ power penalty was observed. And this penalty increases further when the input power is increased, that is, transient effect increases.

In a simulation for the relation between input power and transient, more powerful input induced higher excursion values in output packets. For example, an input of the total power of $-2 \mathrm{dBm}$ resulted in $2.8 \mathrm{~dB}$ excursion in BD1 output packet. Larger excursion value induces larger power penalty at receiver. The simulation was conducted by based on ordinary differential equation for gain dynamics in EDFA $[10,11]$. 


$$
P_{s}^{I S}\left\{\tau \frac{d G_{s}(t)}{d t}+G_{s}(t)+A_{s}\right\}=-P_{s}^{i n}(t)\left\{\exp \left(G_{s}(t)\right)-1\right\}-P_{p}^{i n}(t)\left\{\exp \left(\frac{P_{s}^{I S}}{P_{p}^{I S}}\left(A_{s}+G_{s}(t)\right)-A_{p}\right)-1\right\}
$$

where $\tau$ is spontaneous lifetime, $G_{s}(t)$ is the dynamic gain of the signal light, $P_{s}^{i n}(t)\left(P_{p}^{i n}\right), P_{s}^{I S}\left(P_{p}^{I S}\right)$ and $A_{s}\left(A_{p}\right)$ are the optical input power, the intrinsic saturation power and the total absorption constant of the signal light (the pump light) respectively.

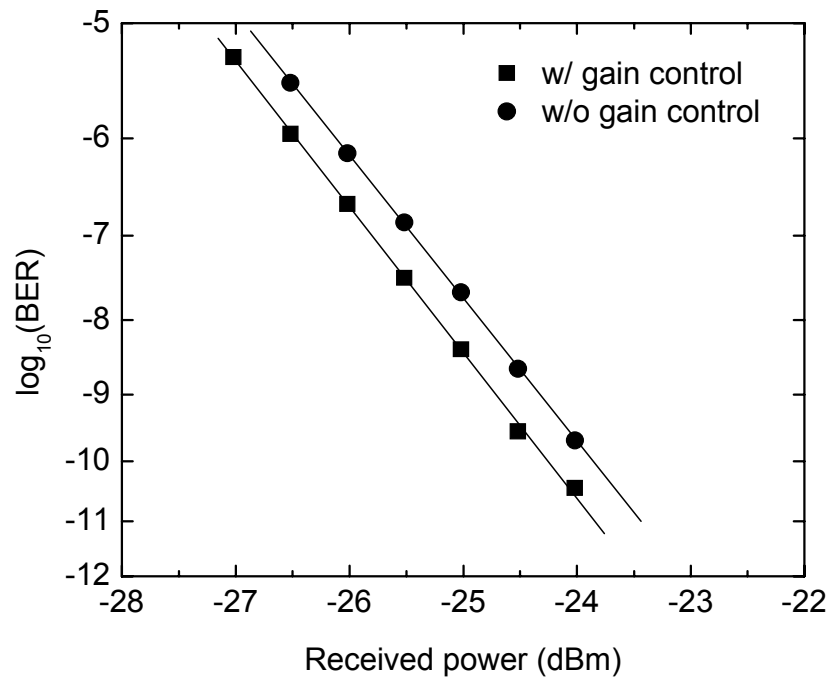

Fig. 6. BER curves of the BD1 channel

\section{CONCLUSION}

We have proposed and experimentally demonstrated a technique for controlling the EDFA gain transient in optical burst network. The transient is induced by slow gain dynamics of the EDFA. A BCP channel power was adjusted based on the information in the BCP. The power excursion and fluctuation of the BD channels were effectively suppressed and $0.4 \mathrm{~dB}$ reduction in power penalty for BD packet was achieved by the proposed technique. The BCP channel did not show severe power penalty even for 0.33 modulation index. The number of $\mathrm{BD}$ channel covered by one $\mathrm{BCP}$ channel and the spacing between the two channels must be carefully considered when the proposed method is applied to OBS system.

\section{ACKNOWLEDGMENT}

This work was supported by the Korean Science and Engineering Foundation (KOSEF) through Optical Internet Research Center (OIRC) project in Information and Communications University.

\section{REFERENCES}

1. C. Qiao, "Labeled Optical Burst Switching for IP-over-WDM Integration," IEEE Comm. Mag. 104-114 (2000). 